\title{
circRNA-002178 act as a ceRNA to promote PDL1/ PD1 expression in lung adenocarcinoma
}

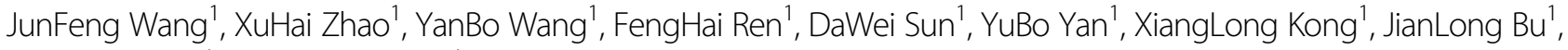
MengFeng Liu' ${ }^{1}$ and ShiDong $\mathrm{Xu}^{1}$

\begin{abstract}
Circular RNAs (circRNAs) have been identified play a vital role in various different types of cancer via sponging miRNAs (microRNAs). However, their role in lung adenocarcinoma (LUAD) remains largely unclear. In this study, we systematically characterized the circRNA expression profiles in the LUAD cancer tissues and paired adjacent noncancerous tissues. Three circRNAs were found to be significantly upregulated. Among them, has-circRNA-002178 was further confirmed to be upregulated in the LUAD tissues, and LUAD cancer cells. Subsequently, we also found hascircRNA-002178 could enhance PDL1 expression via sponging miR-34 in cancer cells to induce T-cell exhaustion. More importantly, circRNA-002178 could be detected in exosomes of plasma from LUAD patients and could serve as biomarkers for LUAD early diagnosis. Finally, we found circRNA-002178 could be delivered into CD8 ${ }^{+} \mathrm{T}$ cells to induce PD1 expression via exosomes. Taken together, our study revealed that circRNA-002178 could act as a ceRNA to promote PDL1/PD1 expression in lung adenocarcinoma.
\end{abstract}

\section{Introduction}

Lung cancer is the leading cause of cancer-related death worldwide $^{1}$. According to histologic types and prognosis, lung adenocarcinoma (LUAD) accounts for $\sim 50 \%$ of all types of lung cancer and is increasing year by year, especially in women and young adults ${ }^{1}$. Despite the improvements of diagnosis and treatment, the 5-year survival rate for LUAD is $<20 \%{ }^{1}$. Therefore, it is urgently needed to find novel early diagnosis biomarkers and treatment targets for LUAD.

More than $90 \%$ human transcripts are found limited protein-coding capacity, but encoded non-coding RNA, including microRNA (miRNA), long non-coding RNA (lncRNA), and circular RNA (circRNA) ${ }^{2}$. CircRNAs are derived from precursor mRNA back-splicing with a circular covalently closed structure and higher tolerance to exonuclease digestion ${ }^{3}$. During the past few years, thousands of circRNAs have been identified in various

Correspondence: ShiDong Xu (xusd163@163.com)

${ }^{1}$ Harbin Medical University Cancer Hospital, Harbin, Heilongjiang, China

These authors contributed equally: JunFeng Wang, XuHai Zhao

Edited by S. Inoue organisms and found play a key role in many diseases, especially cancer ${ }^{3}$. Accumulating evidence is now unveiling that circRNAs could act as ceRNA by sponging miRNA to relieve the repression of miRNAs for their targets. Exosomes are cell-derived vesicles with diameters of $30-100 \mathrm{~nm}$. Studies have confirmed exosomes are present in many body fluids, including blood, urine, and saliva ${ }^{4}$. CircRNA have been found to be stably present in exosomes, and could serve as novel non-invasive biomarkers ${ }^{5}$. More interestingly, these circRNAs present in the exosomes could act as messengers in cell-to-cell communications to regulate the function of target cells ${ }^{6}$.

Tumors cells could grow and metastasize by avoiding recognition and attack by the immune system (which is named tumor immune escape) through various mechanisms, such as the expression of Programmed death-ligand 1 (PDL1, also called CD274, B7-H1) ${ }^{7}$. PDL1, 40-kDa transmembrane protein, has been found to be abundantly expressed in almost all kinds of cancer cells and involved in tumor immune escape through the interaction with programmed cell death protein $1(\mathrm{PD} 1)^{8}$. PD1, encoded by the PDCD1 gene, is a type I transmembrane protein 
composed of 288 amino acid residues, belonging to the immunoglobulin CD28 family. Studies have confirmed PD1 can activate intracellular signaling pathways to inhibit the activation of immune cells or exhaust the immune cell, especially activated $\mathrm{T}$ cells in tumor condition to help cancer cells escape T-cell-mediated death and resist anti-tumor immune responses ${ }^{8}$. Blocking the PDL1/ PD1 pathway is recognized as a very promising approach for tumor treatment of tumor ${ }^{8}$. However, the upstream regulatory mechanism of PDL1 and PD1 still remains largely unknown.

In this study, the circRNAs of LUAD cancer tissues and paired adjacent non-cancerous tissues was characterized by circRNA microarray profile and quantitative reverse transcription PCR (qRT-PCR). CircRNA-002178 was found to be upregulated in LUAD tissues. Subsequently, we found has-circRNA-002178 could enhance PDL1 expression via sponging miR-34 in cancer cells by function assay. Moreover, we also found CircRNA-002178 exist in exosomes of plasma from LUAD patients and could serve as biomarkers for LUAD early diagnosis. Additionally, CircRNA-002178 in the exosomes could be delivered into $\mathrm{T}$ cells to promote PD1 expression via sequestering miR-28-5p. In conclusion, our results suggested that has-circRNA-002178 could be used as a potential non-invasive biomarker for the early detection of LUAD, and function as a ceRNA to enhance PDL1 and $\mathrm{PD} 1$ expression in cancer cells and $\mathrm{T}$ cells, respectively.

\section{Methods}

\section{Patients' characteristics}

The expression profile of circRNAs in LUAD was downloaded from the GEO database (GSE101684 and GSE101586). The 105 pairs of LUAD tissues and adjacent non-cancerous tissues were collected from patients who were diagnosed with LUAD at the Harbin Medical University Cancer Hospital (Harbin, China). The serum samples from 30 healthy volunteers and 120 LUAD patients without any treatment were collected at the Harbin Medical University Cancer Hospital (Harbin, China). The detailed clinicopathological features are described in Table 1. The written informed consent was obtained from all patients. The study was authorized by the Ethics Committee of the Harbin Medical University Cancer Hospital and conducted in conformity to the Declaration of Helsinki.

\section{Cell culture}

Human LUAD cell lines A549, PC9, and 95D, and normal human bronchial epithelial cell line BEAS-2B were purchased from American Type Culture Collection (ATCC) (Manassas, VA, USA). BEAS-2B was cultured in RPMI 1640 medium supplemented with $10 \%$ fetal bovine serum (FBS) (HyClone, Logan, UT, USA), penicillin
Table 1 Patient characteristics and clinical features of LUAD tissues.

\begin{tabular}{|c|c|c|c|c|}
\hline \multirow[t]{2}{*}{ Variable } & \multicolumn{3}{|l|}{ Plasma } & \multirow{2}{*}{$\begin{array}{l}\text { Tissue } \\
\text { LUAD } \\
(n=105)\end{array}$} \\
\hline & & $\begin{array}{l}\text { LUAD } \\
(n=120)\end{array}$ & $\begin{array}{l}\text { Normal } \\
(n=30)\end{array}$ & \\
\hline Average age (years) & & $61.3 \pm 7.9$ & $62.7 \pm 4.6$ & $61.2 \pm 8.5$ \\
\hline \multirow[t]{2}{*}{ Gender } & Female & 64 & 15 & 63 \\
\hline & Male & 56 & 15 & 42 \\
\hline \multirow[t]{4}{*}{ Stage } & 1 & 60 & & 27 \\
\hline & $\|$ & 30 & & 41 \\
\hline & III & 25 & & 37 \\
\hline & IV & 5 & & 0 \\
\hline
\end{tabular}

$(10,000$ units $/ \mathrm{ml})$, and streptomycin $(10,000 \mu \mathrm{g} / \mathrm{ml})$ (Invitrogen). A549, PC9, and 95D were cultured in Dulbecco's Modified Eagle's Medium (DMEM, Gibco, Carlsbad, CA, USA) with 10\% fetal bovine serum (FBS) (HyClone, Logan, UT, USA), penicillin (10,000 units/ml), and streptomycin $(10,000 \mu \mathrm{g} / \mathrm{ml})$ (Invitrogen). All the cells were maintained in a $5 \% \mathrm{CO}_{2}$ humidified atmosphere at $37^{\circ} \mathrm{C}$.

\section{Exosome isolation and incubation with $\mathrm{CD8}^{+} \mathrm{T}$ cells}

To isolate exosomes from serum or cell culture media, the Total Exosome Isolation Kit (from plasma) (Cat\#4484450, Invitrogen, USA) and the Total Exosome Isolation Reagent (from cell culture media) (Cat\# 4478359, Invitrogen, USA) were used following the manufacturer's protocol. For incubation of exosomes with $\mathrm{CD}^{+} \mathrm{T}$ cells, pre-activated $\mathrm{CD} 8^{+} \mathrm{T}$ cells were seeded on 12-well dishes, and $500 \mu \mathrm{g}$ exosomes was added into each well. After incubation for $24 \mathrm{~h}, \mathrm{CD}^{+} \mathrm{T}$ cells were collected for qRT-PCR and the Flow cytometry.

\section{RNA extraction}

Total RNAs were extracted using TRIzol reagent (Takara, Dalian, China) according to the manufacturer's instruction. The nuclear and cytoplasmic fractions were extracted using PARIS Kit (Ambion, Life Technologies). For the RNAs in exosomes isolation, total RNA of the acquired exosome pellet was extracted using the mirVana PARIS Kit (Ambion, Thermo Scientific, Shanghai, China) according to the manufacturer's protocol. Synthetic Caenorhabditis elegans miRNA cel-miR-39 (5'-UCACC GGGUGUAAAUCAGCUUG-3'), (RiboBio, Guangzhou, China) was spiked into the denatured exosomes as a normalization control. The quality and quantity of isolated RNA was detected by Nanodrop 2000 spectrophotometer (Thermo Fisher Scientific, USA). 
Table 2 The primers used for qRT-PCR.

\begin{tabular}{lll}
\hline Genes & Forward & Reverse \\
\hline circRNA- & AGCCCGGGAAGGCGAGACAG & GCTCGGGGGCCCTGTTGG \\
002178 & & \\
RPPH1 & TGGGCAGGAGATGCCGTGGA & CAAAGGAGGCATCCGCCGGG \\
\hline
\end{tabular}

\section{RT-PCR and qRT-PCR}

RNA was reverse transcribed using HiScript II Q RT SuperMixfor qPCR ( + gDNA wiper) (Vazyme, Nanjing, China). The AmpliTaq DNA Polymerase (Life Technologies) was used for PCR. The cDNA and gDNA PCR products were observed using $2 \%$ agarose gel electrophoresis. For qRT-PCR of circRNAs and genes, the AceQ qPCR SYBR Green Master Mix (Vazyme, Nanjing, China) was used. For miRNAs, the Hydrolysis probe-based qRTPCR assay was performed according to the manufacturer's instructions (Applied Biosystems). Primers are listed in Table 2.

\section{RNase $\mathbf{R}$ treatment and actinomycin D assay}

Two micrograms of total RNA was incubated for $30 \mathrm{~min}$ at $37^{\circ} \mathrm{C}$ with or without $5 \mathrm{U} / \mu \mathrm{g}$ RNase $\mathrm{R}$ (Epicenter Technologies). 95D cells were exposed to $2 \mu \mathrm{g} / \mathrm{ml}$ actinomycin D (Sigma) at indicated time point. Then total RNA of these cells was extracted by TRIzol reagent and analyzed using qRT-PCR.

\section{Vector construction and cell transfection}

For luciferase reporter vector, the sequence of circR002178, PDL1 3'UTR, PD1 3'UTR, mutated circR002178, mutated PDL1 3'UTR and PD1 3'UTR was synthesized and then cloned into the downstream of pGL3promoter (Geneseed, Guangzhou, China). The relative luciferase activity was examined by Dual Luciferase Assay Kit (Promega, Madison, WI, USA). Small interfering RNAs (siRNAs) of circR-002178, miR-34a mimics, miR28-5p mimics, and corresponding negative control (NC) were synthesized by GenePharma (Shanghai, China). Cells were transfected using Lipofectamine 3000 (Invitrogen) according to the manufacturer's instruction.

\section{RNA immunoprecipitation (RIP) and biotin-coupled miRNA capture}

The Magna RIP RNA-Binding Protein Immunoprecipitation Kit (Millipore, Billerica, MA) and AGO2 antibody (Argonaute 2 (C34C6) Rabbit mAb \#2897, Cell Signaling Technology, Beverly, MA) were used to RIP experiments according to the manufacturer's instructions. For biotincoupled miRNA capture, The $3^{\prime}$ end biotinylated miR34a, miR-28-5p or control RNA were transfected into 95D cells for $48 \mathrm{~h}$ before harvest. Then $0.5 \mathrm{ml}$ cell lysis buffer
(Invitrogen, USA) wih complete protease inhibitor cocktail (Roche Applied Science, IN) were added into the cell pellets, and incubated on ice for $30 \mathrm{~min}$. The biotincoupled RNA complex was pulled down by incubating the cell lysates with streptavidin-coated magnetic beads (Life Technologies) by centrifugation at $10,000 \times g$ for $20 \mathrm{~min}$.

\section{Western blot analysis}

The total protein of 95D cells were exacted using protein extraction reagent (Thermo Scientific) with a cocktail of proteinase inhibitors (Roche Applied Science, Switzerland). Equal amount of total protein was separated by $10 \%$ sodium dodecyl sulfate-polyacrylamide gel electrophoresis and transferred onto a polyvinylidene fluoride membrane. The membranes were blocked with 5\% skimmed milk powder and incubated with primary antibodies against PDL1(PD-L1 (E1L3N $\left.{ }^{\circledR}\right) \mathrm{XP}^{\circledR}$ Rabbit mAb \#13684, Cell Signaling Technology, Beverly, MA, USA) and GAPDH (GAPDH (D16H11) XP ${ }^{\circledR}$ Rabbit mAb \#5174, Cell Signaling Technology, Beverly, MA, USA) at $4{ }^{\circ} \mathrm{C}$ overnight and then incubated with secondary antibodies (Anti-rabbit IgG, HRP-linked Antibody \#7074, Cell Signaling Technology, Beverly, MA, USA) at room temperature for $2 \mathrm{~h}$. Finally, the bands were examined by Immobilob $^{\mathrm{TM}}$ Western Chemiluminescent HRP Substrate (Millipore, Billerica, MA, USA).

\section{$\mathrm{CD8}^{+} \mathbf{T}$ isolation}

Human $\mathrm{CD}^{+} \mathrm{T}$ cells purified from healthy donor peripheral blood mononuclearcells (PBMCs) by EasySepTM Direct Human $\mathrm{CD}^{+}$T-Cell Isolation Kit (STEMCELL Technologies). For T-cell activation and proliferation, human $\mathrm{CD}^{+} \mathrm{T}$ cells were added to antiCD3/anti-CD28 antibody (BD Biosciences) pre-bound 24well plates for $48 \mathrm{~h}$. The human T-cell transfection kit (Lonza) was used to transfected the miRNA mimic or scramble RNA into activated $\mathrm{CD}^{+} \mathrm{T}$ cell.

\section{Flow cytometry}

Flow cytometry was performed using a CytoFLEX S (Beckman Coulter Life Sciences, Mississauga, ON). Human $\mathrm{CD}^{+} \mathrm{T}$ cell were stained with $0.2 \mu \mathrm{g}$ of PE antiPD1 (PE Mouse anti-Human CD279 (PD-1) Clone EH12.1, BD Pharmingen) antibody.

\section{Expression and statistical analyses}

Adequate sample size was determined according to the previous studies that performed analogous experiments. A two-sided test was applied. The raw data applying $t$-test was normally distributed. The variance was similar between the groups that are being statistically compared. Data are expressed throughout the manuscript as mean $\pm \mathrm{SD}$. The SPSS 18.0 software was performed to the statistical analyses, 
and the GraphPad Prism 6.0 (GraphPad Software, San Diego, CA, USA) was used to generate the graphs. The Mann-Whitney $U$-test was used to compare significant differences in exosomal circRNA expression between the LUAD patients and healthy volunteers. Receiver operating characteristic curve (ROC) analysis was utilized to estimate the diagnostic value of exosomal circRNA. A $P$-value $<.0 .05$ was regarded as statistically significant.

\section{Results}

\section{Characterization of circRNAs in LUAD tissues}

Microarray data were collected from two studies on circRNA expression in LUAD vs. paired adjacent noncancerous tissues. Two-hundred twenty-six circRNAs were identified to be highly expressed in LUAD in the study performed by Zhao et al. ${ }^{9}$ (Fig. 1a), and 18 circRNAs were identified to be highly expressed in LUAD in the study performed by Chen et al. ${ }^{10}$ (Fig. 1b). Three circRNAs, including circRNA-002178, circRNA-104939, and circRNA-104499 were found to be highly expressed in both studies (Fig. 1c). By qRT-PCR assay, only circRNA002178 was unregulated in LUAD tissues compared to adjacent normal tissues (Fig. 1d).

\section{CircRNA-002178 was highly expressed in LUAD}

CircRNA-002178 derived from a long non-coding RNA, named RPPH1 (Ribonuclease P RNA Component H1) located at chr14:20811436-20811534 with a length of $98 \mathrm{nt}$ (Fig. 2a). We further investigated the expression level of circRNA-002178 in 20 pairs of LUAD tissues by qRT-PCR. In accordance with the data before, the results showed circRNA-002178 were markedly upregulated in LUAD tissues compared with adjacent non-tumor tissues (Fig. 2b). Then, we also observed the expression of circRNA-002178 in three kinds of LUAD cancer cell line (A549, PC9, and 95D) and normal human bronchial epithelial cell line (BEAS-2B). As expected, circRNA-002178 was much higher in cancer cell lines, compared to the normal cell line (Fig. 2c). Subsequently, we performed RT-PCR after RNase R treatment to check the resistance of the circRNA-002178 to RNase R digestion. The results showed only circRNA-002178 was resistant to RNase R treatment compared to RPPH1 linear isoform (Fig. 2d). The stability of circRNA-002178 and RPPH1 were further analysed in 95D cells treated with Actinomycin D, an inhibitor of transcription. As previously reported ${ }^{3}$, We found the circRNA-002178 was extremely more stable
A

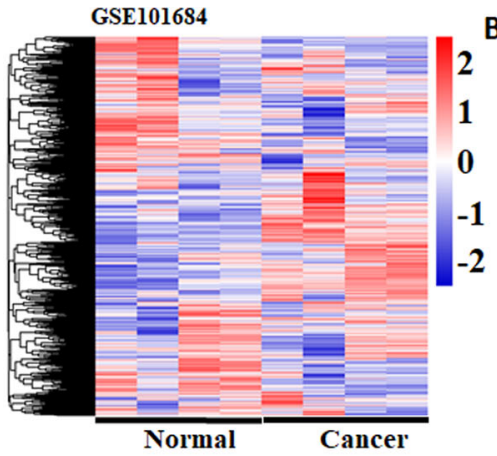

C

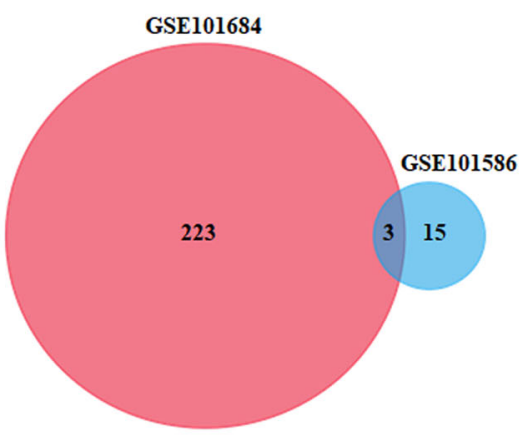

D
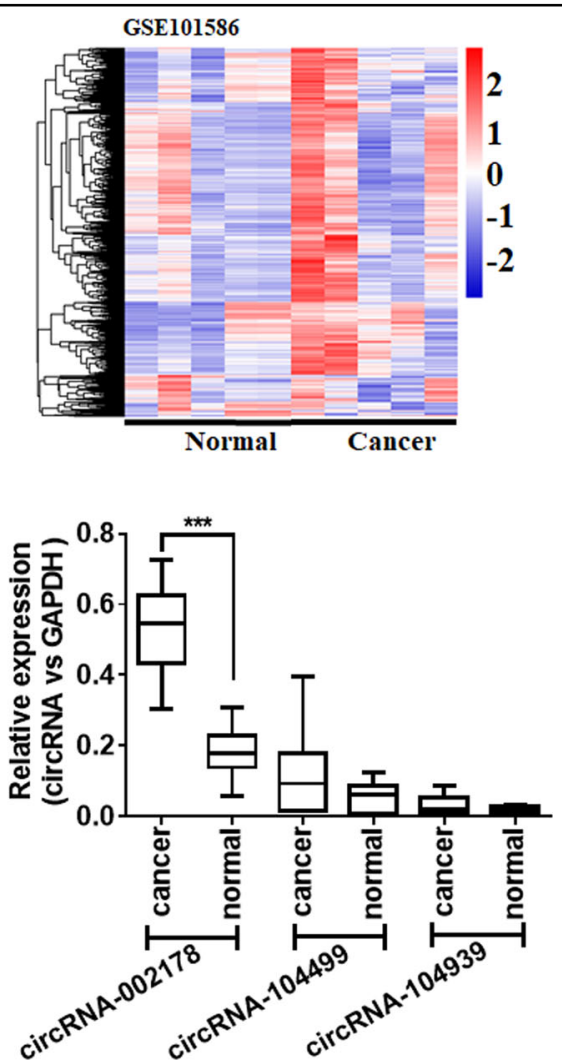

Fig. 1 circRNA expression profiles in LUAD. $\mathbf{a}$, $\mathbf{b}$ The heatmap of circRNA profiles in lung adenocarcinoma tissues (Can) and normal lung tissues (Nor). c The Venn diagram. d The expression level of circRNA-002178, circR-104939, and circRNA-104499 in 20 LUAD cancer tissues and paired adjacent non-cancerous tissues. ${ }^{* *} P<0.001$ as determined by two-tailed $t$-test. 

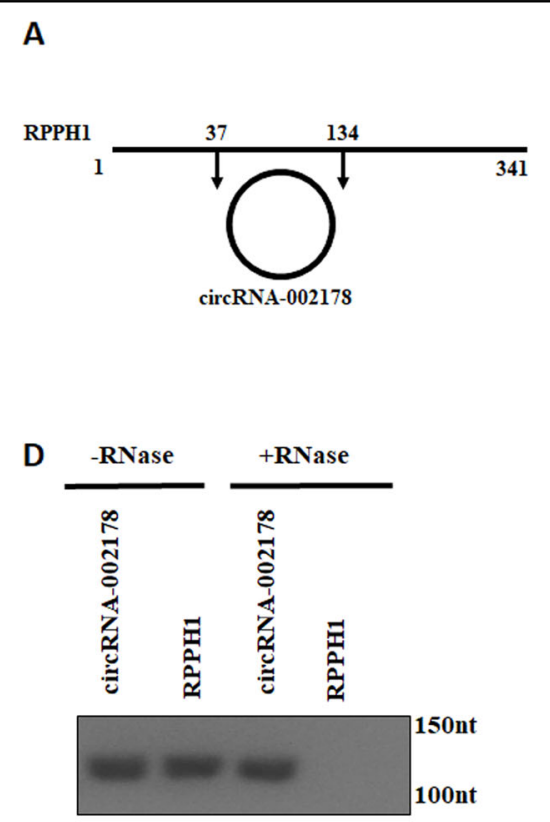

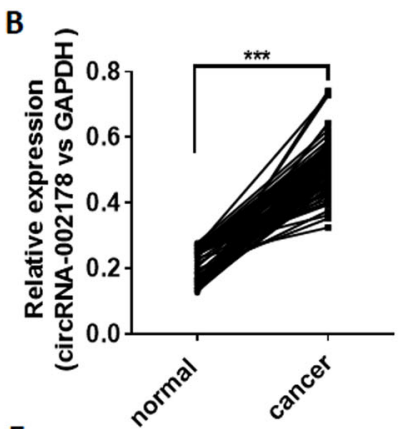

E

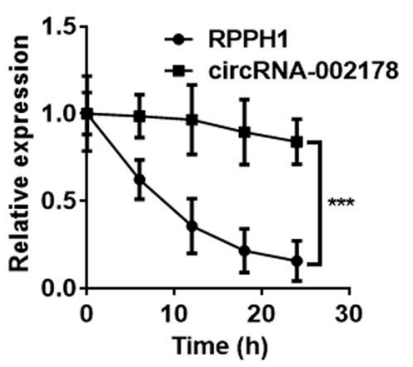

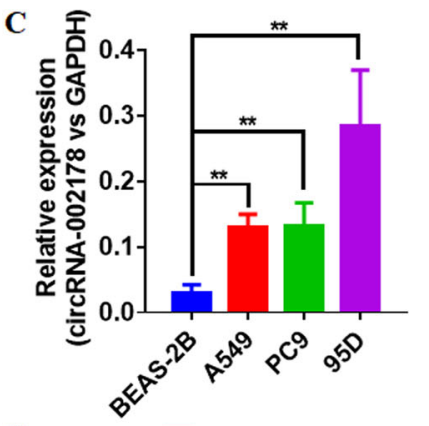

$\mathbf{F}$

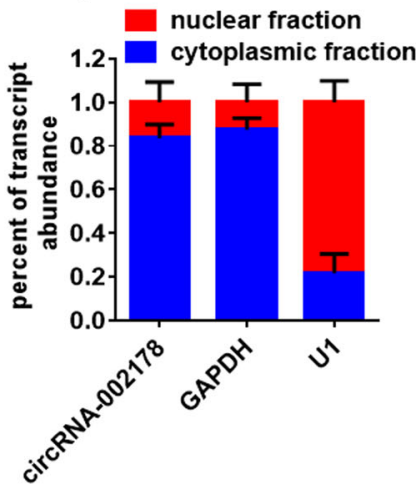

Fig. 2 circRNA-002178 was significantly unregulated in LUAD. a Genomic loci of circRNA-002178 gene. circRNA-002178 is produced at the RPPH1 gene locus. $\mathbf{b}$ qRT-PCR for the abundance of circRNA-002178 in 85 LUAD cancer tissues (Cancer) and paired adjacent non-cancerous tissues (Normal). c qRT-PCR for the abundance of circRNA-002178 in normal lung cell line BESA-2B and LUAD cancer cell line A549, PC9 and 95D. d PCR analysis for circRNA-002178 in CDNA in 95D cells treated with RNase or without. e qRT-PCR for the abundance of circRNA-002178 and RPPH1 in 95D cells treated with Actinomycin D at the indicated time point. $\mathbf{f}$ Levels of circRNA-002178 in the nuclear and cytoplasmic fractions of $95 \mathrm{D}$ cells. ${ }^{*} P<$ 0.01 and ${ }^{* *} P<0.001$ as determined by two-tailed $t$-test.

than RPPH1 (Fig. 2e). To observe the cellular localization of circRNA-002178, the nuclear and cytoplasmic fraction were isolated by PARIS Kit (Ambion, Life Technologies). As showed in Fig. 2f, circRNA-002178 transcript was preferentially located in the cytoplasm (Fig. 2f).

\section{circRNA-002178 enhance PDL1 expression via absorbing miR-34a}

CircRNA can act as a ceRNA to absorb microRNA and indirectly stimulate the protein expression of the target genes of miRNAs ${ }^{11}$. As the circRNA-002178 has been found to be remarkably upregulated in LUAD tissues, the miRNAs downregulated in the LUAD tissues were choosesed to predict the potential binding sites in the circRNA-002178 by miRanda ${ }^{12}$ and RNAhydrid ${ }^{13}$. We analyzed the miRNA expression profiles of the LUAD tissues and non-tumor tissues in the TCGA database, and found 30 miRNAs showed significantly downregulated in LUAD cancer tissues (Table. S1). Among these $30 \mathrm{miR}$ NAs, miR-30c-3p, miR-133a-3p, and miR-34a showed to have at least one binding site with circRNA-002178. We further detected the expression level of these three miRNAs in 20 pairs of LUAD tissues by qRT-PCR. Only miR34a showed significantly decreased in LUAD tissues (Fig.
S1). As predicted by miRanda and RNAhydrid, miR-34a have three binding sites with circRNA-002178 (Fig. 3a). To validate the binding capability of the miRNAs to circRNA-002178, the circRNA-002178 luciferase reporter system was constructed and transfected with miR-34a mimics or scramble RNA into 95D cells. The results showed miR-34a significantly reduced the luciferase activity, while has no influence on the mutated vector, which has no binding site of miR-34a (Fig. 3b). Argonaute 2 (AGO2), the key component of RNA-induced silencing complex (RISC), is the major protein in the regulation of miRNA for its target gene. We pulled down the RNA transcripts, which bind to AGO2 with anti-AGO2 antibody by the anti-AGO2 RNA immunoprecipitation (RIP) assay in 95D cells. Compared to the IgG, circRNA-002178 and miR-34a were significantly enrich in the AGP2 pulldown complex (Fig. 3c). These results showed circRNA002178 could bind with miR-34a in tumor cells. As expected, the miR-34a remarkably increased when the circRNA-002178 was knockdowned by the specific circRNA-002178 siRNAs (Fig. 3d and S2A), while this increase could be abolished by co-transfected with miR34a inhibitor, which could inhibit miR-34a (Fig. 3h and Fig. S2C). Since it is well known that miR-34a could 


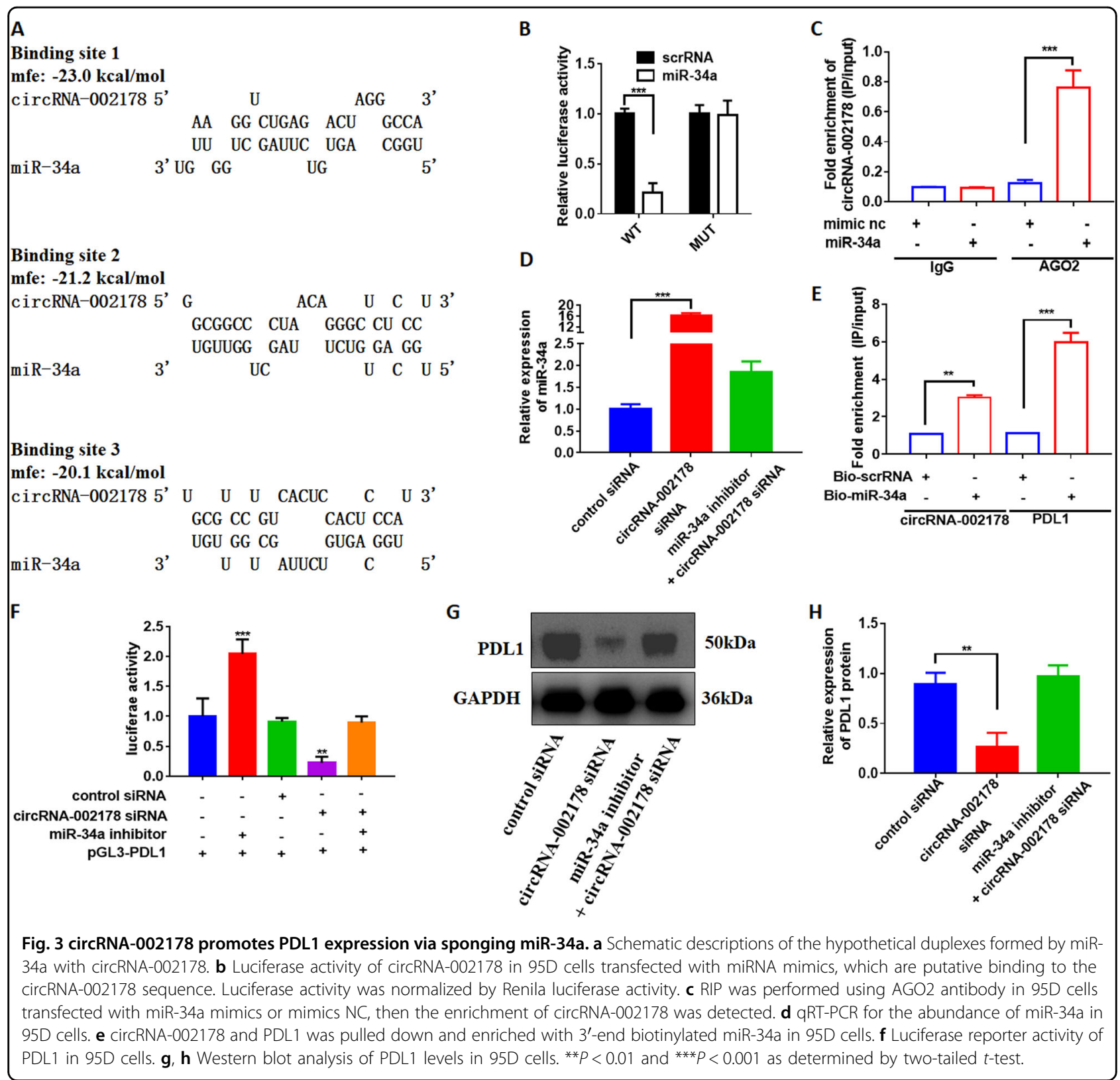

repress PDL1 expression in cancer cells ${ }^{14}$. We found knockdown miR-34a by miR-34a inhibitor could upregulate PDL1 expression (Fig. S2D). The miRNA pulldown assay with specific biotin-labeled miR-34a also showed circRNA-002178 and PDL1 was specific enriched in the biotin-labeled miR-34a group compared with control (Fig. 3e). Additionally, the activity of luciferase reporter vector carrying the PDL1 $13^{\prime}$ UTR sequence could be significantly decreased by knockdown circRNA002178 through siRNAs, while this decrease could be attenuated by miR-34a inhibitor (Fig. 3f). Finally, we evaluated the protein expression of PDL1 in 95D cells transfected with circRNA-002178 siRNA. Result showed that knockdown circRNA-002178 by siRNA significantly decreased PDL1 expression (Fig. 3g, h). As miR-34a inhibitor could suppress the increase of miR-34a caused by the circRNA-002178 siRNA (Fig. 3d), the decrease of PDL1 induced by circRNA-002178 siRNA could be abolished by miR-34a inhibitor (Fig. 3g, h). Taken together, our result suggested circRNA-002178 could enhance PDL1 expression via absorbing miR-34a in cancer cells.

circRNA-002178 existed in exosomes and serve as a novel diagnosis biomarker for LUAD

Exosomes, secreted by cancer cells, have been found to contain lots of RNAs, including miRNA, IncRNA, and 

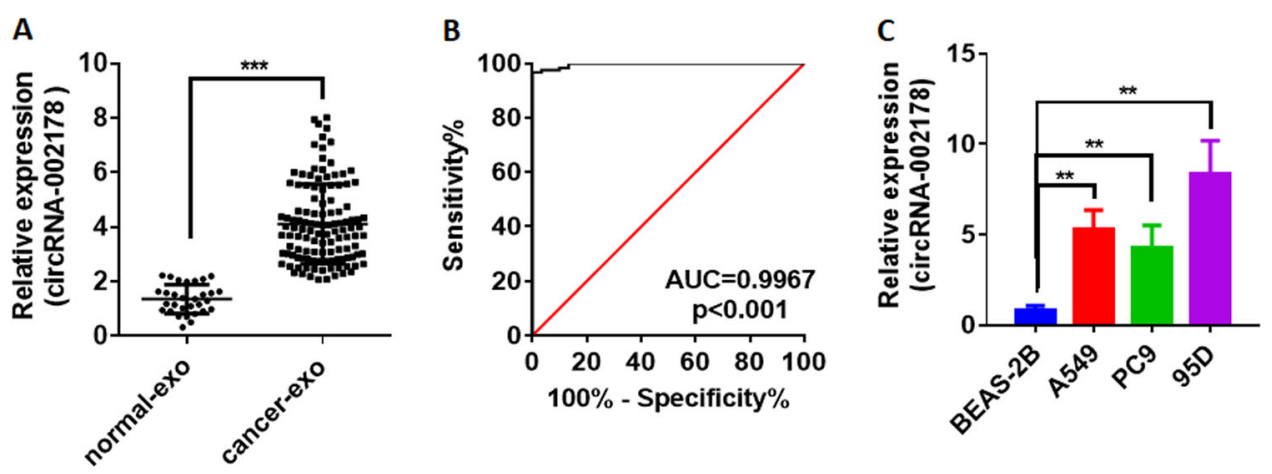

Fig. 4 circRNA-002178 existed in exosomes and serve as a novel diagnosis biomarker for LUAD. a The expression of circRNA-002178 in the serum exosomes from patients with LUAD $(N=120)$ and normal samples from healthy volunteers $(N=30)$. b ROC analysis for the circRNA-002178. c the expression of circRNA-002178 in the exosomes derived from normal lung cell line BESA-2B and LUAD cancer cell line A549, PC9, and 95D. ${ }^{* *} P<0.01$ and ${ }^{* * *} P<0.001$ as determined by two-tailed $t$-test.
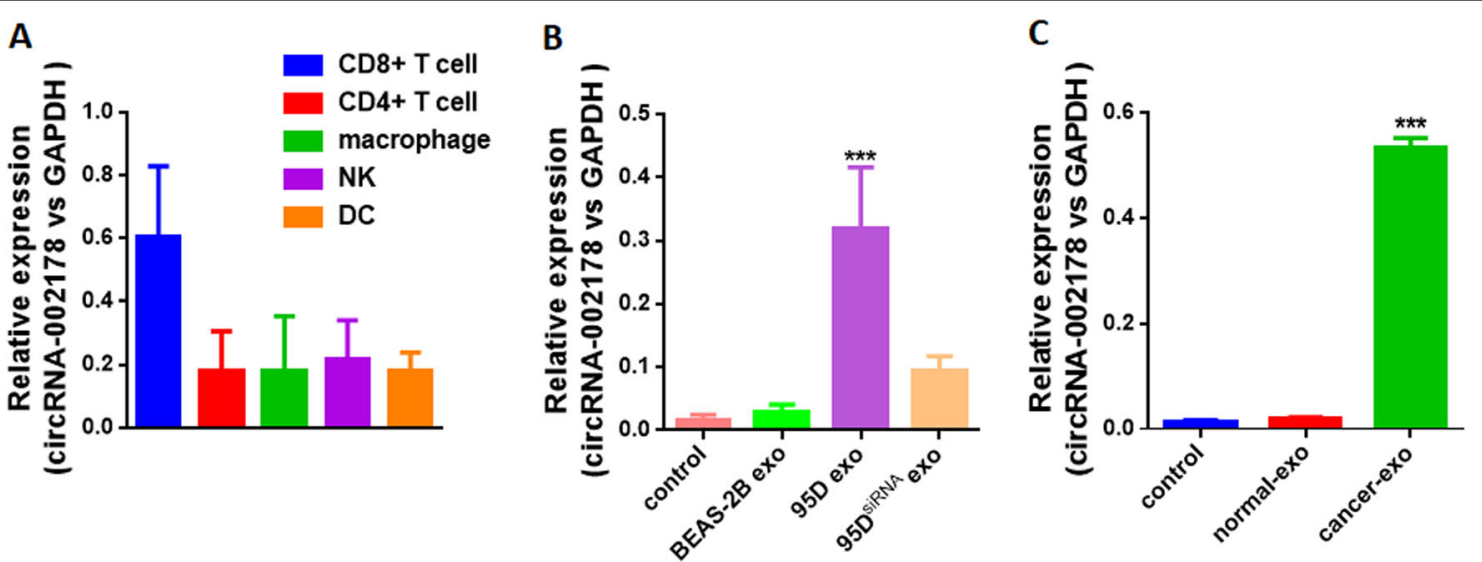

Fig. 5 circRNA- 002178 could be delivered into $\mathrm{CDB}^{+} \mathbf{T}$ cells via exosomes secreted by cancer cells. a The expression of circRNA-002178 in the immune cells derived from normal tissues or lung cancer tissues. $\mathbf{b}$ The expression of circRNA-002178 in the CD8 ${ }^{+} \mathrm{T}$ cells incubated with exosomes derived from BEAS-2B, 95D, or 95D transfected with circRNA-002178 siRNAs. $\mathbf{c}$ The expression of circRNA-002178 in the CD8 ${ }^{+}$T cells incubated with exosomes derived from healthy volunteer serum or LUAD patient serum. Each value represents the mean $\pm S D$; ${ }^{* *} P<0.001$ as determined by twotailed t-test.

circRNA ${ }^{6}$. We isolated exosomes in the serum from healthy volunteers or LUAD patients, and performed qRT-PCR to detect the circRNA-002178 in the exosomes. The results showed circRNA-002178 was significantly increased in exosomes from the LUAD patients compared to the healthy volunteers $(P<0.001$, Fig. 4a). The areas under the curve (AUCs) of the circRNA-002178 were $0.9956(P<0.001$, Fig. 4b). As the results in the patients, we also found the circRNA-002178 was much higher in the exosomes derived from cancer cells than the exosomes derived from normal human bronchial epithelial cells (Fig. 4c). These results reaffirmed that serum exosomal circRNA-002178 could serve as a novel diagnosis biomarker for LUAD. However, this needs to be further studied in the big cohort.
circRNA-002178 promoted PD1 expression via absorbing miR-28-5p in $\mathrm{CD}^{+}{ }^{+} \mathrm{T}$ cells

In the tumor microenvironment, crosstalk of cancer cells with immune cells is essential for tumor immune invasion $^{15}$. Exosomes derived from cancer cells are involved in tumor immune escape by delivering RNAs (such as miRNAs, circRNAs) into immune cells ${ }^{6,16}$. In order to identify the function of circRNA-002178 in exosomes during the tumor immune escape, we firstly detected the circRNA-002178 expression level in the immune cells isolated from cancer tissues, and found circRNA-002178 significantly enriched in the $\mathrm{CD}^{+}$ $\mathrm{T}$ cells (Fig. 5a). Thus, these results suggested circRNA002178 could be transferred from cancer cells into $\mathrm{D} 8^{+}$ $\mathrm{T}$ cells via exosomes. Then we isolated the exosomes from 


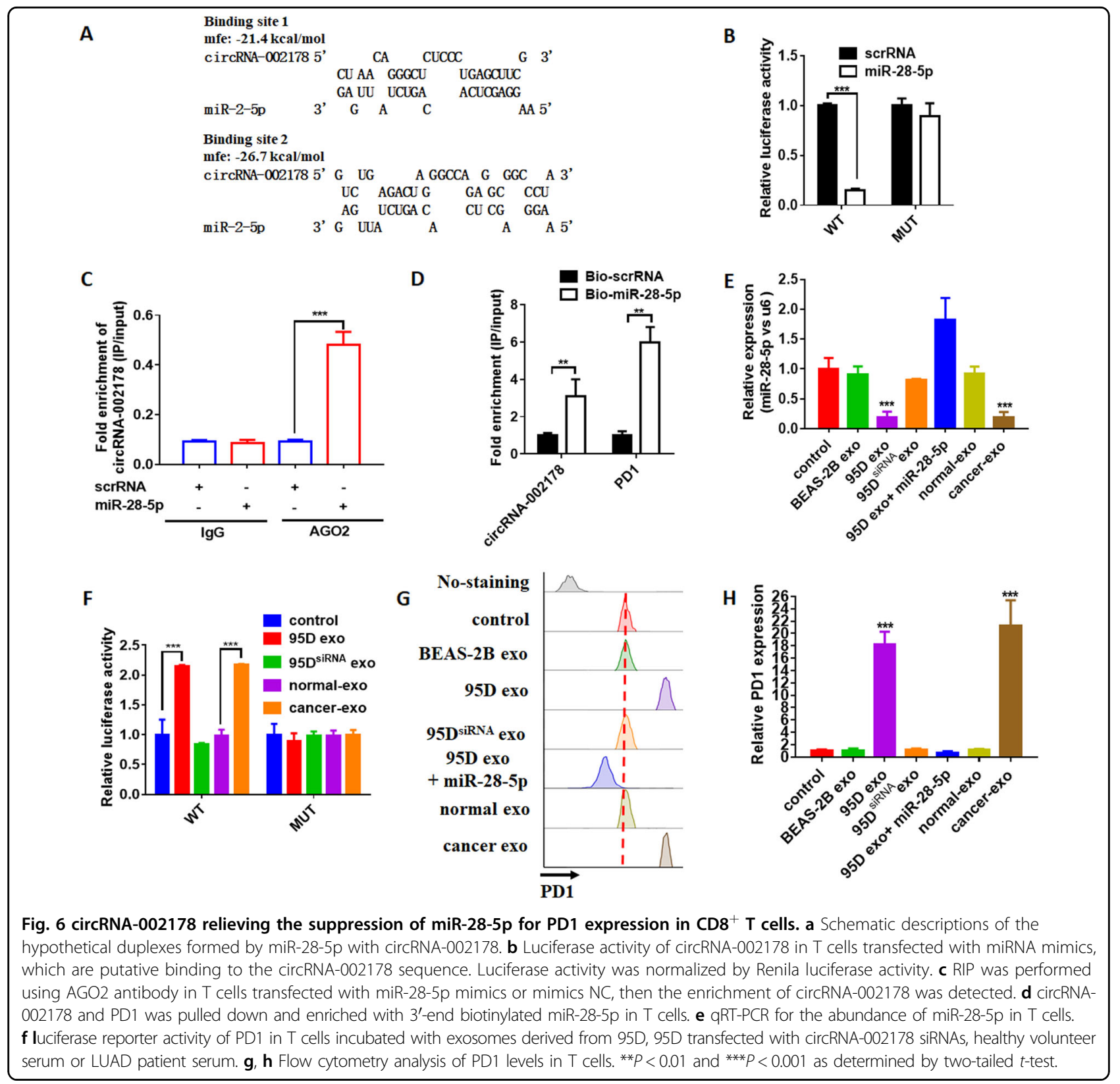

the culture media of LUAD cancer cell line 95D or normal human bronchial epithelial cell line BEAS-2B, and incubated the exosomes with pre-activated $\mathrm{CD}^{+}{ }^{+} \mathrm{T}$ cells. As showed in Fig. 5b, the circRNA-002178 markedly increased in the $\mathrm{CD}^{+} \mathrm{T}$ cells incubated with the exosomes from 95D cells, compared to the $\mathrm{CD}^{+} \mathrm{T}$ cells incubated with the exosomes from BEAS-2B cells (Fig. $5 b)$. Additionally, when the circRNA-002178 in the exosomes derived from 95D cells was knockdowned by the siRNA (Fig. S2B), the circRNA-002178 was found not to be upregulated in the $\mathrm{CD}^{+} \mathrm{T}$ cells (Fig. 5b). More interestingly, the exosomes derived from cancer patient serum could also significantly increase the expression level of circRNA-002178 in $\mathrm{CD}^{+} \mathrm{T}$ cells, compared to the exosomes from serum of healthy volunteers (Fig. 5c). In summary, these result showed circRNA-002178 in the exosomes secreted by cancer cells could be transferred into $\mathrm{CD}^{+} \mathrm{T}$ cells.

As previously mentioned, circRNAs usually regulate the gene expression by acting as miRNA sponges. We use the miRanda and RNAhydrid to predicted the binding site of circRNA-002178 and the dysregulated miRNAs in the activated $\mathrm{CD} 8^{+} \mathrm{T}$ cells ${ }^{17,18}$. The result showed miR-28-5p have two binding sites in the circRNA-002178 (Fig. 6a). In order to confirm the bioinformatics prediction, we transfected the full-length of circRNA-002178-wt and mutant (without miR-28-5p binding sites) luciferase reporter vector pGL3 into $\mathrm{CD} 8^{+} \mathrm{T}$ cells with miR-28-5p 
mimic or scrRNA. The result showed miR-28-5p mimics could significantly downregulated the luciferase activity of WT group but not mutant one (Fig. 5b). Then, we performed the anti-AGO2 RNA immunoprecipitation (RIP) assay to pull down the RNA transcripts, which bind to AGO2 with anti-AGO2 antibody in $\mathrm{CD}^{+} \mathrm{T}$ cells. As showed in Fig. 6c, circRNA-002178 and miR-28-5p were all efficiently pulled down by anti-AGO2 antibodies compared with IgG. These results suggested there might be a direct interaction between circRNA-002178 and miR-28-5p. It has been reported miR-28-5p could repress PD1 expression by binding the $3^{\prime} \mathrm{UTR}$ of PD1 in CD8 ${ }^{+}$ $\mathrm{T}$ cells ${ }^{19}$. As the previous reported ${ }^{19}$, we found miR-28-5p could repress the luciferase activity of luciferase reporter vector pGL3 containing the PD1 3'UTR sequence (Fig $\mathrm{S} 3 \mathrm{~B}$ ), and repress the expression of PD1 in $\mathrm{CD}^{+} \mathrm{T}$ cells (Fig. S3C, D). Subsequently, the biotin-labeled miR-28-5p was transfected into $\mathrm{CD}^{+}{ }^{+} \mathrm{T}$ cells to perform the miRNA pull-down assay. The results showed a specific enrichment of circRNA-002178 and PD1 in the biotin-labeled miR-28-5p group compared with control (Fig. 6d). Then, we incubated the exosomes derived from 95D cells or BEAS-2B cells with $\mathrm{CD}^{+} \mathrm{T}$ cells. As the exosomes secreted from 95D cells and serum of LUAD patients could increase the expression level of circRNA-002178 in $\mathrm{CD}^{+} \mathrm{T}$ cells, we found miR-28-5p significantly decreased in $\mathrm{CD}^{+} \mathrm{T}$ cells incubated with these exosomes, compared to the exosomes from BEAS-2B cells and serum of healthy volunteers (Fig. 6e), and the decrease could be attenuated by miR-28-5p mimic (Fig. 6e). Immediately, the luciferase reporter vector pGL3 with the PD1 3'UTR sequence or mutated sequence were transfected into $\mathrm{CD}^{+} \mathrm{T}$ cells, which later incubated with the exosomes. As showed in Fig. 6f, the exosomes from 95D cells or serum of LUAD patients could significantly downregulated the luciferase activity, while the exsomes form 95D cells transfected with circRNA-002178 siRNAs has no effect. Finally, we evaluated the protein expression of $\mathrm{PD} 1$ in $\mathrm{CD}^{+} \mathrm{T}$ cells. Result showed that the PD1 level significantly increased in $\mathrm{CD}^{+} \mathrm{T}$ cells incubated with exosomes from cell media of 95D cells or serum of LUAD patients, compared to the exosomes from BEAS-2B cells, or 95D cells transfected with circRNA-002178 siRNA, or the exosomes from serum of healthy volunteers (Fig. 6g, h). Taken together, our result suggested circRNA-002178 delivered via exosomes derived from cancer cells could promote PD1 expression via absorbing miR-28-5p in $\mathrm{CD} 8^{+} \mathrm{T}$ cells.

\section{Discussion}

LUAD is one of the most common malignant tumors worldwide, and its therapeutic effect is still far from satisfactory because of the lack of effective diagnosis and treatment methods ${ }^{1}$. circRNA is novel kind of non-coding
RNA by noncanonical splicing without a free $3^{\prime}$ or $5^{\prime}$ end ${ }^{3}$. Numbers of studies have revealed circRNAs play a key role in tumor process ${ }^{5}$. In our study, we found a circRNA named circRNA-002178 significantly increased in the tissues and exosomes, and could have potential to serve as a biomarker for LUAD diagnosis. CircRNA-002178 as one of the 27 circRNAs derived from the exon region of the derived from Ribonuclease $\mathrm{P}$ RNA component $\mathrm{H} 1$ (RPPH1) has been reported overexpressed in esophageal cancer cells ${ }^{20}$. By function assay, we found circRNA002178 could act as a sponge for miR-34a and miR-28-5p to relieve the suppression of these two miRNAs for their target genes PDL1 and PD1 in cancer cells and CD8 ${ }^{+}$ $\mathrm{T}$ cells, respectively (Fig. 7).

The T-cell-based immune system has been confirmed to closely involve in the cancer cell reorganization and clearance $^{7}$. This progress is controlled by a series of coinhibitory receptors and their ligands (also known as immune checkpoints), which could result to tumor immune escape ${ }^{7}$. Among all the immune checkpoints, the PDL1/PD1 has been proven as a therapeutic target in a large number of malignancies ${ }^{7}$. Recently, antibodies targeting the PDL1/PD-1 have been successfully approved for cancer treatment, including melanoma, lung cancer, renal cell carcinoma, bladder cancer, head and neck squamous cell carcinoma ${ }^{8}$. However, only a minority of patients was found to benefit from the PDL1/PD1 blockade $^{8}$. Several studies have confirmed the expression of PDL1 on tumor cells has been related to the clinical response. Thus, a better understanding of the processes that regulate PDL1/PD1 expression is urgently needed for PDL1/PD-1 therapy. During the past years, the expression of PDL1 in tumor cells is found to be affected by genomic aberrations, inflammatory signaling, oncogenic signaling, posttranslational modulation and microRNA-based control $^{20}$. MiR-34a is a member of miR-34 family and found to be frequently downregulated and could function as tumor suppressive miRNA in solid malignancies ${ }^{21}$. It could directly bind with the $3^{\prime}$ UTR of PDL1 and inhibit the expression of this immune checkpoint molecule in many tumors, including $\operatorname{LUAD}^{14}$. In our study, we firstly identified a LUAD-specific circRNA, circRNA-002178 could act as sponge for miR-34a to relive the repression of miR-34a for PDL1 and promote PDL1 expression in tumor cells. Activated $\mathrm{T}$ cells were found to be high expressed several miRNAs ${ }^{19}$. Among them, miR-28-5p was revealed to bind with the $3^{\prime} \mathrm{UTR}$ of PD1 to control the expression level of PD1 on T cells ${ }^{19}$. It's well known that tumor cells and tumor microenvironment could induce PD1 expression on activated $\mathrm{T}$ cells to induce $\mathrm{T}$-cell exhaustion. However, the mechanism remains unknown. In our study, we found cancer cells could secrete exosomes, which contains circRNA-002178 and these exosomes could deliver circRNA-002178 into $\mathrm{T}$ cells to 


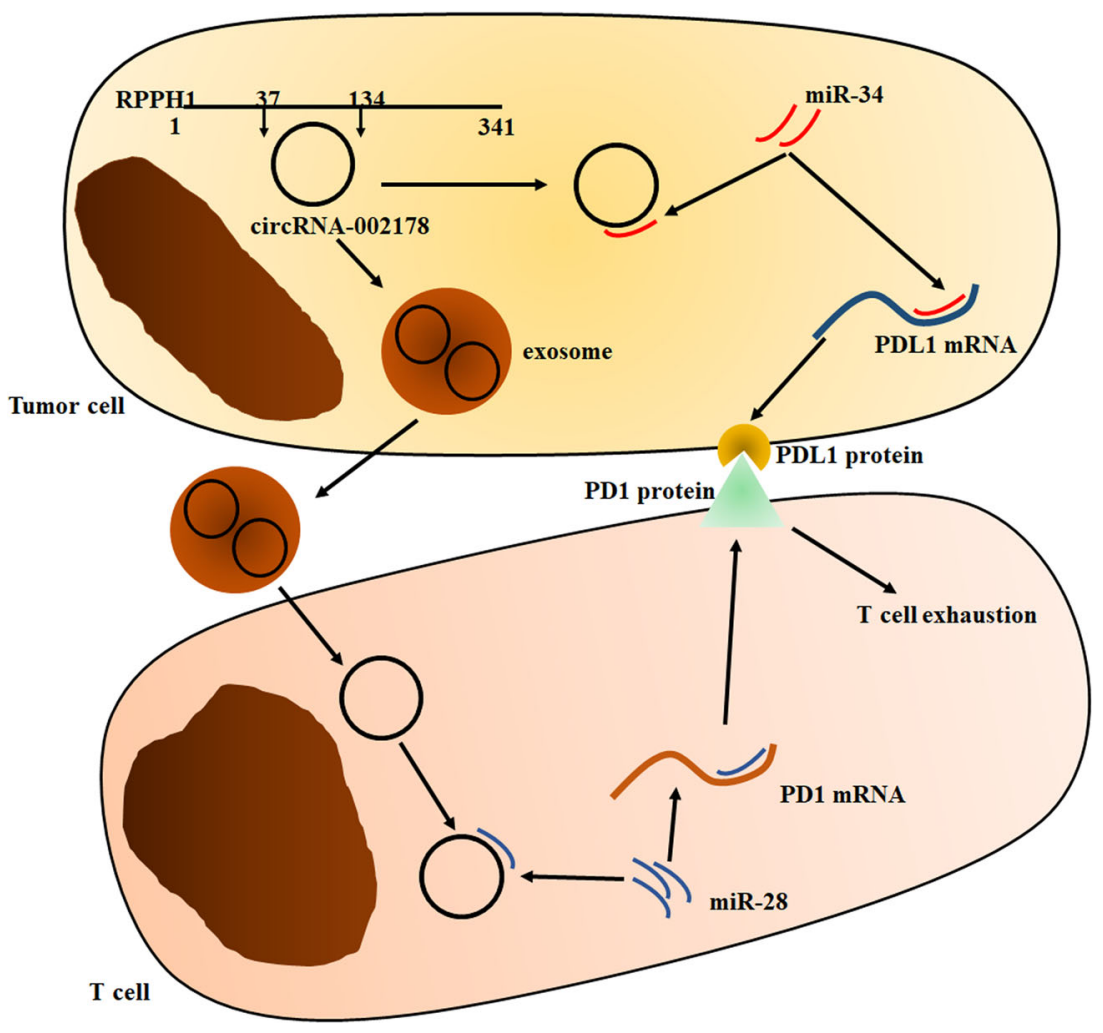

Fig. 7 Hypothesis diagram illustrates function and mechanism of circRNA-002178 in LUAD progress.

enhance PD1 expression by sponging the miR-28-5p. As we know, this is the first study to reveal the circRNA derived from tumor cells, which could simultaneously regulate PDL1 and PD1 in tumor cells and T cells via acting as a sponge for miRNAs, respectively (Fig. 7).

To conclude, we found circRNA-002178 was significantly upregulated in the LUAD tissues, cells and exosomes of cancer cells and serum from patients. circRNA-002178 could enhance PDL1 expression via sponging miR-34 in cancer cells. Moreover, circRNA002178 could also be delivered into $\mathrm{T}$ cells to promote PD1 expression via sequestering miR-28-5p through exosomes secreted by cancer cells. Additionally, the exosomal circRNA-002178 significantly upregulated in the serum from LUAD patients. Our results suggested that circRNA-002178 could be used as a potential noninvasive biomarker for the LUAD detection and could act as a target of immune therapy since it could promote the expression of PDL1 and PD1 expression.

\section{Acknowledgements}

This work was supported by grants from Special Fund for Scientific and Technological Innovation Talents Research of Harbin Science and Technology Bureau and Nn10 Program of Harbin Medical University Cancer Hospital.
Data availability

All data generated or analyzed during this study are included in this published article. Raw and processed data are stored in the laboratory of global data bank (GB) and are available upon request.

Conflict of interest

The authors declare that they have no conflict of interest.

\section{Ethical approval}

This study was approved by the Ethics Committee of Harbin Medical University Cancer Hospital.

\section{Informed consent}

Informed consent was obtained from all patients. All experiments were performed in accordance with relevant guidelines and regulations.

\section{Publisher's note}

Springer Nature remains neutral with regard to jurisdictional claims in published maps and institutional affiliations.

Supplementary Information accompanies this paper at (https://doi.org/ 10.1038/s41419-020-2230-9).

Received: 5 August 2019 Revised: 31 December 2019 Accepted: 2 January 2020

Published online: 16 January 2020

\footnotetext{
References

1. Siegel, R. L., Miller, K. D. \& Jemal, A. Cancer statistics, 2019. CA Cancer J. Clin. 69 7-34 (2019).
} 
2. Salmena, L., Poliseno, L., Tay, Y., Kats, L. \& Pandolfi, P. P. A ceRNA hypothesis: the Rosetta Stone of a hidden RNA language? Cell 146, 353-358 (2011)

3. Li, X., Yang, L. \& Chen, L. L. The biogenesis, functions, and challenges of circular RNAs. Mol. Cell 71, 428-442 (2018).

4. $\mathrm{Xu}, \mathrm{L} ., \mathrm{Wu}, \mathrm{L}$. F. \& Den, F. Y. Exosome: an emerging source of biomarkers for human diseases. Curr. Mol. Med. 19, 387-394 (2019).

5. Shang, Q., Yang, Z., Jia, R. \& Ge, S. The novel roles of circRNAs in human cancer. Mol. Cancer 18, 6 (2019).

6. Wang, Y. et al. Exosomal circRNAs: biogenesis, effect and application in human diseases. Mol. Cancer 18, 116 (2019).

7. Jiang, X. J. et al. Immunotherapy targeted to immune checkpoint: a revolutionary breakthrough in cancer therapy. Prog. Biochem. Biophys. 45, 1178-1186 (2018).

8. Jiang, $X$. et al. Role of the tumor microenvironment in PD-L1/PD-1-mediated tumor immune escape. Mol. Cancer 18, 10 (2019).

9. Zhao, J. et al. CircRNA expression profile in early-stage lung adenocarcinoma patients. Cell Physiol. Biochem. 44, 2138-2146 (2017).

10. Chen, T. et al. Circ_0078767 suppresses non-small-cell lung cancer by protecting RASSF1A expression via sponging miR-330-3p. Cell Prolif. 52, e12548 (2019).

11. Zhong, Y. X. et al. Circular RNAs function as ceRNAs to regulate and control human cancer progression. Mol. Cancer 17, 79 (2018).
12. Betel, D., Wilson, M., Gabow, A., Marks, D. S. \& Sander, C. The microRNA.org resource: targets and expression. Nucleic Acids Res. 36, D149-D153 (2008).

13. Kruger, J. \& Rehmsmeier, M. RNAhybrid: microRNA target prediction easy, fast and flexible. Nucleic Acids Res. 34, W451-W454 (2006).

14. Cortez, M. A. et al. PDL1 Regulation by p53 via miR-34. J. Natl Cancer Inst. 108 1-9 (2016).

15. Yang, H., Sun, L. \& Mao, Y. The role of exosomes in tumor immunity. Ann. Transl. Med 6, S116 (2018)

16. Liu, Y., Gu, Y. \& Cao, X. The exosomes in tumor immunity. Oncoimmunology 4, e1027472 (2015).

17. Wallaert, A. et al. Comprehensive miRNA expression profiling in human T-cell acute lymphoblastic leukemia by small RNA-sequencing. Sci. Rep. 7, 7901 (2017)

18. Ghisi, M. et al. Modulation of microRNA expression in human T-cell development: targeting of NOTCH3 by miR-150. Blood 117, 7053-7062 (2011).

19. Li, Q. et al. miR-28 modulates exhaustive differentiation of $T$ cells through silencing programmed cell death-1 and regulating cytokine secretion. Oncotarget 7, 53735-53750 (2016).

20. Su, H. F. et al. Profiling and bioinformatics analyses reveal differential circular RNA expression in radioresistant esophageal cancer cells. J. Transl. Med. 14, 225 (2016).

21. Smolle, M. A., Calin, H. N., Pichler, M. \& Calin, G. A. Noncoding RNAs and immune checkpoints-clinical implications as cancer therapeutics. FEBS J. 284 , 1952-1966 (2017). 